\title{
Quantization of Two-Dimensional Gravity with Dynamical Torsion
}

\author{
P. M. Lavrov*, P. Yu. Moshin \\ Tomsk State Pedagogical University, Tomsk 634041, Russia
}

\begin{abstract}
We consider two-dimensional gravity with dynamical torsion in the BV and BLT formalisms of gauge theories quantization as well as in the background field method.
\end{abstract}

\section{Introduction}

Two-dimensional theories of gravity ${ }^{1-9}$ and superagrvity ${ }^{10-12}$ are interesting mainly due to their close relation to string and superstring theory. On the other hand, simple two-dimensional models provide a deeper insight into classical and quantum properties of gravity in higher dimensions. It should also be noted that in a number of cases such models are exactly solvable at the classical level.

One of the models of two-dimensional gravity which has been extensively discussed recently (at both the classical ${ }^{13-16}$ and quantum ${ }^{17-20}$ levels) is the model ${ }^{7}$ originally proposed in the context of bosonic string theory with dynamical torsion ${ }^{21}$ in order to address certain difficulties of string theory. Thus, the study of Ref. 21 showed (using the path integral approach) that there is no critical dimension for the string with dynamical torsion. Note also that the model ${ }^{7}$ presents the most general theory of two-dimensional $R^{2}$-gravity with independent dynamical torsion that leads to second-order equations of motion for the zweibein and Lorentz connection. The equations of motion of the model have been analyzed in Refs. 7, 13, 14 (in conformal 7,13 and light-cone ${ }^{14}$ gauges) where their complete integrability was demonstrated.

At the same time, the model ${ }^{7}$ contains solutions with constant curvature and zero torsion, thus incorporating a number of other two-dimensional gravity models ${ }^{1,2,9}$ (whose actions, however, do not admit, as compared to that of Ref. 7, of purely geometric interpretation).

The Hamiltonian structure of gauge symmetries of the model ${ }^{7}$ has been studied in Ref. 16 and its canonical quantization, in Refs. 17, 18.

Our interest in the theory of two-dimensional gravity with dynamical torsion ${ }^{7}$ is due to the recent paper $^{20}$ whose authors made an attempt at quantizing a model (with auxiliary fields) they suggested, which is classically equivalent to that of Ref. 7. To quantize this theory (in its formulation with the algebra of gauge transformations open of-shell) the authors ${ }^{20}$ use a modification of the Faddeev-Popov rules; however, in our opinion, they failed to give a consistent procedure for constructing the quantum action. Notice that the classical equivalence of two theories generally does not imply their equivalence at the quantum level (see, for example, Ref. [22]). In this connection, the present paper deals with the treatment of the original model ${ }^{7}$ in different versions of Lagrangian quantization, namely in the $\mathrm{BV}^{23}$ and $\mathrm{BLT}^{24}$ quantization schemes for general gauge theories as well as in the background field method (for details see, for example, Ref. 25).

We use De Witt's condensed notations ${ }^{26}$ in general formulas of Refs. 23, 24. The Grassmann parity and ghost number of a certain quantity $A$ are denoted $\varepsilon(A), \operatorname{gh}(A)$ respectively.

*E-mail: lavrov@tspi.tomsk.su 
For indices of quantities transforming by the Lorentz group, we use Latin characters: $i$, $j, k \ldots(i=0,1) ; \varepsilon^{i j}$ is a constant antisymmetric second-rank pseudo-tensor subject to the normalization condition $\varepsilon^{01}=1$. Greek characters stand for indices of quantities transforming as (pseudo-)tensors under the general coordinates transformations: $\lambda, \mu, \nu \ldots(\lambda=0,1)$; given this $\epsilon^{\mu \nu}=-\epsilon^{\nu \mu}\left(\epsilon^{01}=1\right)$. Derivatives with respect to fields are understood as the right-hand, and those with respect to sources and antifields, as the left-hand ones.

\section{Two-dimensional Gravity with Dynamical Torsion}

The theory ${ }^{7}$ of two-dimensional gravity with dynamical torsion is described in terms of the zweibein and Lorentz connection $\left(e_{\mu}^{i}, \omega_{\mu}\right)$ by the action

$$
\mathcal{S}\left(e_{\mu}^{i}, \omega_{\mu}\right)=\int d^{2} x e\left(\frac{1}{16 \alpha} R_{\mu \nu}^{i j} R^{\mu \nu}{ }_{i j}-\frac{1}{8 \beta} T_{\mu \nu}{ }^{i} T_{i}^{\mu \nu}-\gamma\right)
$$

where $\alpha, \beta, \gamma$ are constant parameters. In Eq. (1), the Latin indices are lowered with the help of the Minkowski metric $\eta_{i j}(+,-)$, and the Greek indices, with the help of the metric tensor $g_{\mu \nu}=\eta_{i j} e_{\mu}^{i} e_{\nu}^{j}$. Besides, the following notations are used:

$$
\begin{aligned}
e & =\operatorname{det} e_{\mu}^{i}, \\
R_{\mu \nu}{ }^{i j} & =\varepsilon^{i j} R_{\mu \nu}, \quad R_{\mu \nu}=\partial_{\mu} \omega_{\nu}-(\mu \leftrightarrow \nu), \\
T_{\mu \nu}{ }^{i} & =\partial_{\mu} e_{\nu}^{i}+\varepsilon^{i j} \omega_{\mu} e_{\nu j}-(\mu \leftrightarrow \nu) .
\end{aligned}
$$

The action (1) is invariant under the local Lorentz transformations $e_{\mu}^{i} \rightarrow e_{\mu}^{i}, \omega_{\mu} \rightarrow \omega_{\mu}^{\prime}$

$$
\begin{aligned}
e_{\mu}^{i} & =\left(\Lambda e_{\mu}\right)^{i}, \\
\left(\Omega_{\mu}^{\prime}\right)_{j}^{i} & =\left(\Lambda \Omega_{\mu} \Lambda^{-1}\right)_{j}^{i}+\left(\Lambda \partial_{\mu} \Lambda^{-1}\right)_{j}^{i}, \quad\left(\Omega_{\mu}\right)_{j}^{i}=\varepsilon^{i k} \eta_{k j} \omega_{\mu},
\end{aligned}
$$

or infinitesimally (with the parameter $\zeta$ )

$$
\delta e_{\mu}^{i}=\varepsilon^{i j} e_{\mu j} \zeta, \quad \delta \omega_{\mu}=-\partial_{\mu} \zeta,
$$

as well as under the general coordinates transformations

$$
\begin{aligned}
x & \rightarrow x^{\prime}=x^{\prime}(x), \\
e_{\mu}^{i} & \rightarrow e_{\mu}^{\prime i}\left(x^{\prime}\right)=\frac{\partial x^{\lambda}}{\partial x^{\prime} \mu} e_{\lambda}^{i}(x), \\
\omega_{\mu} & \rightarrow \omega_{\mu}^{\prime}\left(x^{\prime}\right)=\frac{\partial x^{\lambda}}{\partial x^{\prime} \mu} \omega_{\lambda}(x),
\end{aligned}
$$

and, consequently, under the corresponding infinitesimal transformations (with the parameters $\left.\xi^{\mu}\right)$

$$
\begin{aligned}
\delta e_{\mu}^{i} & =e_{\nu}^{i} \partial_{\mu} \xi^{\nu}+\left(\partial_{\nu} e_{\mu}^{i}\right) \xi^{\nu} \\
\delta \omega_{\mu} & =\omega_{\nu} \partial_{\mu} \xi^{\nu}+\left(\partial_{\nu} \omega_{\mu}\right) \xi^{\nu} .
\end{aligned}
$$

Added together, the gauge transformations (3), (5) form a closed algebra

$$
\begin{aligned}
{\left[\delta_{\zeta(1)}, \delta_{\zeta(2)}\right] } & =0 \\
{\left[\delta_{\xi(1)}, \delta_{\xi(2)}\right] } & =\delta_{\xi(1,2)} \\
{\left[\delta_{\zeta}, \delta_{\xi}\right] } & =\delta_{\zeta^{\prime}}
\end{aligned}
$$


where

$$
\xi_{(1,2)}^{\mu}=\xi_{(1)}^{\nu} \partial_{\nu} \xi^{\mu}{ }_{(2)}-\left(\partial_{\nu} \xi^{\mu}{ }_{(1)}\right) \xi_{(2)}^{\nu}, \quad \zeta^{\prime}=\left(\partial_{\mu} \zeta\right) \xi^{\mu} .
$$

The action (1) (when $e \neq 0$ ) admits of the representation

$$
\mathcal{S}\left(e_{\mu}^{i}, \omega_{\mu}\right)=\int d^{2} x\left(\frac{1}{4 e \alpha} F^{2}+\frac{1}{4 e \beta} T_{i} T^{i}-e \gamma\right),
$$

where

$$
F=\frac{1}{2} \epsilon^{\mu \nu} R_{\mu \nu}, \quad T^{i}=\frac{1}{2} \epsilon^{\mu \nu} T_{\mu \nu}^{i}
$$

and is equivalent ${ }^{20}$ to the action $\tilde{\mathcal{S}}=\tilde{\mathcal{S}}\left(e_{\mu}^{i}, \omega_{\mu}, \varphi, \varphi_{i}\right)$

$$
\tilde{\mathcal{S}}\left(e_{\mu}^{i}, \omega_{\mu}, \varphi, \varphi_{i}\right)=\int d^{2} x\left\{\left(\varphi F+\varphi_{i} T^{i}\right)-e\left(\alpha \varphi^{2}+\beta \varphi_{i} \varphi^{i}+\gamma\right)\right\}
$$

after the auxiliary fields $\varphi, \varphi_{i}$ have been eliminated with the help of the equations of motion of $\tilde{\mathcal{S}}$

$$
\begin{aligned}
\frac{\delta \tilde{\mathcal{S}}}{\delta e_{\mu}^{i}} & =\epsilon^{\mu \nu}\left\{\partial_{\nu} \varphi_{i}+\varepsilon_{i j} \omega_{\nu} \varphi^{j}+\varepsilon_{i j} e_{\nu}^{j}\left(\alpha \varphi^{2}+\beta \varphi_{k} \varphi^{k}+\gamma\right)\right\} \\
\frac{\delta \tilde{\mathcal{S}}}{\delta \omega_{\mu}} & =\epsilon^{\mu \nu}\left(\partial_{\nu} \varphi+\varepsilon^{i j} \varphi_{i} e_{\nu j}\right) \\
\frac{\delta \tilde{\mathcal{S}}}{\delta \varphi} & =F-2 \alpha e \varphi \\
\frac{\delta \tilde{\mathcal{S}}}{\delta \varphi_{i}} & =T^{i}-2 \beta e \varphi^{i}
\end{aligned}
$$

The action (7) is invariant under the complex of the local Lorentz transformations (2) of the initial fields $e_{\mu}^{i}, \omega_{\mu}$ and the transformations of the auxiliary fields $\varphi, \varphi_{i}$

$$
\varphi^{\prime}=\varphi, \quad \varphi_{i}^{\prime}=\left(\Lambda^{-1}\right)_{i}^{j} \varphi_{j}
$$

as well as under the complex of the general coordinates transformations (4) and transformations of the form

$$
\varphi^{\prime}\left(x^{\prime}\right)=\varphi(x), \quad \varphi_{i}^{\prime}\left(x^{\prime}\right)=\varphi_{i}(x) .
$$

Infinitesimally, (2), (8) and (4), (9) imply the gauge transformations (with the parameters $\zeta$, $\left.\xi^{\mu}\right)$

$$
\begin{aligned}
\delta e_{\mu}^{i} & =\varepsilon^{i j} e_{\mu j} \zeta+e_{\nu}^{i} \partial_{\mu} \xi^{\nu}+\left(\partial_{\nu} e_{\mu}^{i}\right) \xi^{\nu} \\
\delta \omega_{\mu} & =-\partial_{\mu} \zeta+\omega_{\nu} \partial_{\mu} \xi^{\nu}+\left(\partial_{\nu} \omega_{\mu}\right) \xi^{\nu}, \\
\delta \varphi & =\left(\partial_{\mu} \varphi\right) \xi^{\mu} \\
\delta \varphi_{i} & =\varepsilon_{i j} \varphi^{j} \zeta+\left(\partial_{\nu} \varphi_{i}\right) \xi^{\nu},
\end{aligned}
$$

which form a closed algebra of the form (6).

Notice that the action (7) is also invariant under the infinitesimal transformations (with the parameters $\left.\bar{\zeta}, \bar{\xi}^{i}\right)$

$$
\begin{aligned}
\bar{\delta} e_{\mu}^{i} & =\varepsilon^{i j} e_{\mu j} \bar{\zeta}+\partial_{\mu} \bar{\xi}^{i}+\varepsilon^{i j} \omega_{\mu} \bar{\xi}_{j}+2 \beta \varepsilon_{k l} \varphi^{i} e_{\mu}^{k} \bar{\xi}^{l} \\
\bar{\delta} \omega_{\mu} & =-\partial_{\mu} \bar{\zeta}+2 \alpha \varepsilon_{k l} \varphi e_{\mu}^{k} \bar{\xi}^{l} \\
\bar{\delta} \varphi & =-\varepsilon^{i j} \varphi_{i} \bar{\xi}_{j} \\
\bar{\delta} \varphi_{i} & =\varepsilon_{i j} \varphi^{j} \bar{\zeta}-\varepsilon_{i j}\left(\alpha \varphi^{2}+\beta \varphi_{k} \varphi^{k}+\gamma\right) \bar{\xi}^{j}
\end{aligned}
$$


whose algebra is open on the extremals of $\tilde{\mathcal{S}}(7)$

$$
\begin{aligned}
{\left[\bar{\delta}_{\bar{\zeta}(1)}, \bar{\delta}_{\bar{\zeta}(2)}\right] } & =0 \\
{\left[\bar{\delta}_{\bar{\zeta}}, \bar{\delta}_{\bar{\xi}}\right] } & =\bar{\delta}_{\bar{\xi}^{\prime}}, \\
{\left[\bar{\delta}_{\bar{\xi}(1)}, \bar{\delta}_{\bar{\xi}(2)}\right] } & =\bar{\delta}_{\bar{\zeta}(1,2)}+\bar{\delta}_{\bar{\xi}(1,2)}+2 \epsilon_{\mu \nu} \varepsilon_{k l} \bar{\xi}^{k}{ }_{(1)} \bar{\xi}_{(2)}^{l}\left(\alpha \frac{\delta \mathcal{\mathcal { S }}}{\delta \omega_{\mu}} \frac{\delta}{\delta \omega_{\nu}}+\beta \eta^{i j} \frac{\delta \mathcal{\mathcal { S }}}{\delta e_{\mu}^{i}} \frac{\delta}{\delta e_{\nu}^{j}}\right),
\end{aligned}
$$

where

$$
\begin{aligned}
\bar{\xi}^{i} & =-\varepsilon^{i j} \bar{\zeta}_{j}, \\
\bar{\zeta}_{(1,2)} & =-2 \alpha \varepsilon_{i j} \bar{\xi}^{i}{ }_{(1)} \bar{\xi}^{j}{ }_{(2)} \varphi \\
\bar{\xi}^{i}{ }_{(1,2)} & =2 \beta \varepsilon_{j k} \bar{\xi}^{j}{ }_{(1)} \bar{\xi}^{k}{ }_{(2)} \varphi^{i} .
\end{aligned}
$$

The transformations (11) correspond to a set of generators which is equivalent to that of Eq. (10) and coincide, for the choice of the parameters $\bar{\zeta}=\zeta-\omega_{\mu} \xi^{\mu}, \bar{\xi}^{i}=e_{\mu}^{i} \xi^{\mu}$, with (10) on-shell

$$
\begin{aligned}
\bar{\delta} e_{\mu}^{i} & =\delta e_{\mu}^{i}-\epsilon_{\mu \nu} \frac{\delta \tilde{\mathcal{S}}}{\delta \varphi_{i}} \xi^{\nu} \\
\bar{\delta} \omega_{\mu} & =\delta \omega_{\mu}-\epsilon_{\mu \nu} \frac{\delta \tilde{\mathcal{S}}}{\delta \varphi} \xi^{\nu} \\
\bar{\delta} \varphi & =\delta \varphi+\epsilon_{\mu \nu} \frac{\delta \tilde{\mathcal{S}}}{\delta \omega_{\mu}} \xi^{\nu}, \\
\bar{\delta} \varphi_{i} & =\delta \varphi_{i}+\epsilon_{\mu \nu} \frac{\delta \tilde{\mathcal{S}}}{\delta e_{\mu}^{i}} \xi^{\nu} .
\end{aligned}
$$

Notice that the result of Lagrangian quantization generally depends on the way the initial gauge theory is chosen from the class of equivalent theories and, in particular, on the choice of generators of gauge transformations. Thus, the study of Ref. 22 has demonstrated that a theory with a unitary $S$-matrix for a certain choice of generators may prove non-unitary for another choice. In this connection, we further concentrate on the original model (1), (3), (5), $(6)$.

\section{Quantization of the Model}

Let us consider the original model $(1),(3),(5),(6)$ in the framework of the BV quantization formalism $^{23}$ for irreducible gauge theories. To this end, we first introduce the complete configuration space $\phi^{A}$. It is constructed by extending the initial space of the fields $\left(e_{\mu}^{i}, \omega_{\mu}\right)$ with the help of the Faddeev-Popov ghosts $\left(\bar{C}, C, \bar{C}^{\mu}, C^{\mu}\right)$ and the Lagrangian multipliers $\left(b, b^{\mu}\right)$ according to the number of the gauge parameters in Eqs. (3), (5) (for $\zeta, \xi^{\mu}$ respectively). The Grassmann parity and ghost numbers of the fields $\phi^{A}$

$$
\phi^{A}=\left(e_{\mu}^{i}, \omega_{\mu} ; b, b^{\mu} ; \bar{C}, C, \bar{C}^{\mu}, C^{\mu}\right)
$$

have the form

$$
\begin{aligned}
\varepsilon\left(e_{\mu}^{i}\right) & =\varepsilon\left(\omega_{\mu}\right)=\varepsilon(b)=\varepsilon\left(b^{\mu}\right)=0, \\
\varepsilon(\bar{C}) & =\varepsilon(C)=\varepsilon\left(\bar{C}^{\mu}\right)=\varepsilon\left(C^{\mu}\right)=1, \\
\operatorname{gh}\left(e_{\mu}^{i}\right) & =\operatorname{gh}\left(\omega_{\mu}\right)=\operatorname{gh}(b)=\operatorname{gh}\left(b^{\mu}\right)=0, \\
\operatorname{gh}(\bar{C}) & =\operatorname{gh}\left(\bar{C}^{\mu}\right)=-1, \quad \operatorname{gh}(C)=\operatorname{gh}\left(C^{\mu}\right)=1 .
\end{aligned}
$$


Besides, we introduce for the fields $\phi^{A}$ the set of the antifields $\phi_{A}^{*}$

$$
\phi_{A}^{*}=\left(e_{i}^{* \mu}, \omega^{* \mu} ; b^{*}, b_{\mu}^{*} ; \bar{C}^{*}, C^{*}, \bar{C}_{\mu}^{*}, C_{\mu}^{*}\right)
$$

with the following distribution of the Grassmann parity and ghost number:

$$
\varepsilon\left(\phi_{A}^{*}\right)=\varepsilon\left(\phi^{A}\right)+1, \quad \operatorname{gh}\left(\phi_{A}^{*}\right)=-1-\operatorname{gh}\left(\phi^{A}\right) .
$$

As is well-known, the generating functional $Z(J)$ of Green's functions for the fields $\phi^{A}$ can be represented, within the BV quantization, in the form of the following functional integral:

$$
Z(J)=\int d \phi d \phi^{*} d \lambda \exp \left\{\frac{i}{\hbar}\left[S\left(\phi, \phi^{*}\right)+\left(\phi_{A}^{*}-\frac{\delta \Psi}{\delta \phi^{A}}\right) \lambda^{A}+J_{A} \phi^{A}\right]\right\} .
$$

In Eq. (12), $J_{A}$ are the sources to the fields $\phi^{A}$

$$
\varepsilon\left(J_{A}\right)=\varepsilon\left(\phi^{A}\right), \quad \operatorname{gh}\left(J_{A}\right)=-\operatorname{gh}\left(\phi^{A}\right),
$$

$\lambda^{A}$ are auxiliary fields

$$
\varepsilon\left(\lambda^{A}\right)=\varepsilon\left(\phi_{A}^{*}\right), \quad \operatorname{gh}\left(\lambda^{A}\right)=-\operatorname{gh}\left(\phi_{A}^{*}\right),
$$

$\Psi=\Psi(\phi)$ is the gauge fermion, and $S=S\left(\phi, \phi^{*}\right)$ is a boson functional satisfying the generating equation

$$
\frac{\delta S}{\delta \phi^{A}} \frac{\delta S}{\delta \phi_{A}^{*}}=i \hbar \frac{\delta^{2} S}{\delta \phi^{A} \delta \phi_{A}^{*}}
$$

with the boundary condition

$$
\left.S\right|_{\phi^{*}=\hbar=0}=\mathcal{S}
$$

where $\mathcal{S}$ is the initial classical action.

The solution of the generating equation (13) satisfying the boundary condition (14) for the model (1), (3), (5), (6) can be represented as a functional $S=S\left(\phi, \phi^{*}\right)$ linear in the antifields (we assume a regularization of dimensional type)

$$
\begin{aligned}
S & =S_{\min }+\int d^{2} x\left(\bar{C}^{*} b+\bar{C}_{\mu}^{*} b^{\mu}\right), \\
S_{\min } & =\mathcal{S}+\int d^{2} x\left\{e_{i}^{* \mu} X_{1 \mu}^{i}+\omega^{* \mu} X_{1 \mu}+C^{*} X_{2}+C_{\mu}^{*} X_{2}^{\mu}\right\},
\end{aligned}
$$

where

$$
\begin{aligned}
X_{1 \mu}^{i} & =\varepsilon^{i j} e_{\mu j} C+\left(\partial_{\lambda} e_{\mu}^{i}\right) C^{\lambda}+e_{\lambda}^{i} \partial_{\mu} C^{\lambda}, \\
X_{1 \mu} & =-\partial_{\mu} C+\left(\partial_{\lambda} \omega_{\mu}\right) C^{\lambda}+\omega_{\lambda} \partial_{\mu} C^{\lambda} \\
X_{2} & =\frac{1}{2}\left(\partial_{\mu} C\right) C^{\mu}, \quad X_{2}^{\mu}=C^{\lambda} \partial_{\lambda} C^{\mu}
\end{aligned}
$$

We choose the gauge fermion $\Psi=\Psi(\phi)$ in the form

$$
\begin{aligned}
& \Psi=\int d^{2} x\left(\bar{C} \chi+\bar{C}^{\mu} \chi_{\mu}\right) \\
& \chi=\eta^{\mu \nu} \partial_{\mu} \omega_{\nu}, \quad \chi_{\mu}=\eta^{\lambda \nu} e_{\lambda i} \partial_{\mu} e_{\nu}^{i}
\end{aligned}
$$

where $\eta^{\mu \nu}(+,-)$ is the metric of the two-dimensional Minkowski space.

By virtue of Eqs. (15), (16), integrating over the variables $\lambda^{A}, \phi_{A}^{*}$ in Eq. (12) yields the following representation of the generating functional of Green's functions:

$$
Z(J)=\int d \phi \exp \left\{\frac{i}{\hbar}\left(\mathcal{S}+S_{\mathrm{GF}}(\phi)+S_{\mathrm{GH}}(\phi)+J_{A} \phi^{A}\right)\right\}
$$


where

$$
\begin{aligned}
S_{\mathrm{GF}}= & \int d^{2} x\left(\chi b+\chi_{\mu} b^{\mu}\right) \\
S_{\mathrm{GH}}= & \int d^{2} x\left\{\eta^{\mu \nu} \partial_{\nu} \bar{C}\left(\partial_{\mu} C-\left(\partial_{\lambda} \omega_{\mu}\right) C^{\lambda}-\omega_{\lambda} \partial_{\mu} C^{\lambda}\right)-\right. \\
& \left.-\left(\eta^{\mu \nu} \bar{C}^{\lambda} \partial_{\lambda} e_{\nu}^{i}-\eta^{\lambda \mu} \partial_{\nu}\left(\bar{C}^{\nu} e_{\lambda}^{i}\right)\right)\left(\varepsilon_{i j} e_{\mu}^{j} C-\left(\partial_{\sigma} e_{\mu i}\right) C^{\sigma}-e_{\sigma i} \partial_{\mu} C^{\sigma}\right)\right\} .
\end{aligned}
$$

Consider now the model (1), (3), (5), (6) in the Lagrangian version of the BLT quantization ${ }^{24}$ of gauge theories. Notice that the Faddeev-Popov ghosts are then combined into the $S p(2)$ doublets $(a=1,2)$

$$
C^{a}=(\bar{C}, C), \quad C^{\mu a}=\left(\bar{C}^{\mu}, C^{\mu}\right),
$$

and the fields $\phi^{A}$ of the complete configuration space are supplemented by the sets of the antifields $\phi_{A a}^{*}, \bar{\phi}_{A}$

$$
\begin{aligned}
\phi_{A a}^{*} & =\left(e_{i a}^{* \mu}, \omega_{a}^{* \mu} ; b_{a}^{*}, b_{\mu a}^{*} ; C_{b a}^{*}, C_{\mu b a}^{*}\right), \\
\bar{\phi}_{A} & =\left(\bar{e}_{i}^{\mu}, \bar{\omega}^{\mu} ; \bar{b}, \bar{b}_{\mu} ; \bar{C}_{a}, \bar{C}_{\mu a}\right)
\end{aligned}
$$

with

$$
\begin{aligned}
\varepsilon\left(\phi_{A a}^{*}\right) & =\varepsilon\left(\phi^{A}\right)+1, \quad \operatorname{gh}\left(\phi_{A a}^{*}\right)=(-1)^{a}-\operatorname{gh}\left(\phi^{A}\right), \\
\varepsilon\left(\bar{\phi}_{A}\right) & =\varepsilon\left(\phi^{A}\right), \quad \operatorname{gh}\left(\bar{\phi}_{A}\right)=-\operatorname{gh}\left(\phi^{A}\right) .
\end{aligned}
$$

The generating functional $Z(J)$ of Green's functions within the Lagrangian version of the BLT method can be represented in the form ${ }^{24}$

$$
\begin{aligned}
Z(J)= & \int d \phi d \phi_{a}^{*} d \bar{\phi} d \pi^{a} d \lambda \exp \left\{\frac { i } { \hbar } \left[S\left(\phi, \phi^{*}, \bar{\phi}\right)+\phi_{A a}^{*} \pi^{A a}+\right.\right. \\
& \left.\left.+\left(\bar{\phi}_{A}-\frac{\delta F}{\delta \phi^{A}}\right) \lambda^{A}-\frac{1}{2} \varepsilon_{a b} \pi^{A a} \frac{\delta^{2} F}{\delta \phi^{A} \delta \phi^{B}} \pi^{B b}+J_{A} \phi^{A}\right]\right\}
\end{aligned}
$$

In Eq. (18), $\varepsilon_{a b}$ is an antisymmetric tensor $\left(\varepsilon_{12}=-1\right) ; \pi^{A a}, \lambda^{A}$ are auxiliary fields

$$
\begin{aligned}
\varepsilon\left(\pi^{A a}\right) & =\varepsilon_{A}+1, \quad \operatorname{gh}\left(\pi^{A a}\right)=-(-1)^{a}+\operatorname{gh}\left(\phi^{A}\right), \\
\varepsilon\left(\lambda^{A}\right) & =\varepsilon_{A}, \quad \operatorname{gh}\left(\lambda^{A}\right)=\operatorname{gh}\left(\phi^{A}\right),
\end{aligned}
$$

$F=F(\phi)$ is the gauge fixing boson functional, and $S=S\left(\phi, \phi^{*}, \bar{\phi}\right)$ is a boson functional satisfying the generating equations of the BLT formalism ${ }^{24}$

$$
\frac{\delta S}{\delta \phi^{A}} \frac{\delta S}{\delta \phi_{A a}^{*}}+\varepsilon^{a b} \phi_{A b}^{*} \frac{\delta S}{\delta \bar{\phi}_{A}}=i \hbar \frac{\delta^{2} S}{\delta \phi^{A} \delta \phi_{A a}^{*}}
$$

with the boundary condition

$$
\left.S\right|_{\phi^{*}=\bar{\phi}=\hbar=0}=\mathcal{S} \text {. }
$$

The solution of the generating equations (19) with the boundary condition (20) for the theory in question can be chosen as a functional linear in the antifields $\phi_{A a}^{*}, \bar{\phi}_{A}$

$$
\begin{aligned}
S=\mathcal{S} & +\int d^{2} x\left\{e_{i a}^{* \mu} X_{1 \mu}^{i a}+\omega_{a}^{* \mu} X_{1 \mu}^{a}+b_{a}^{*} X_{2}^{a}+b_{\mu a}^{*} X_{2}^{\mu a}\right. \\
& \left.+C_{b a}^{*} X_{3}^{a b}+C_{\mu b a}^{*} X_{3}^{\mu a b}+\bar{e}_{i}^{\mu} Y_{1 \mu}^{i}+\bar{\omega}^{\mu} Y_{1 \mu}+\bar{C}_{a} Y_{2}^{a}+\bar{C}_{\mu a} Y_{2}^{\mu a}\right\}
\end{aligned}
$$


where

$$
\begin{aligned}
& X_{1 \mu}^{i a}= \varepsilon^{i j} e_{\mu j} C^{a}+\left(\partial_{\lambda} e_{\mu}^{i}\right) C^{\lambda a}+e_{\lambda}^{i} \partial_{\mu} C^{\lambda a}, \\
& X_{1 \mu}^{a}=-\partial_{\mu} C^{a}+\left(\partial_{\lambda} \omega_{\mu}\right) C^{\lambda a}+\omega_{\lambda} \partial_{\mu} C^{\lambda a}, \\
& X_{2}^{a}= \frac{1}{2}\left(C^{\mu a} \partial_{\mu} b-\frac{1}{6} \varepsilon_{b c} C^{\mu c} \partial_{\mu}\left(C^{\lambda a} \partial_{\lambda} C^{b}\right)\right) \\
& X_{2}^{\mu a}= \frac{1}{2}\left(\left(b^{\lambda} \partial_{\lambda} C^{\mu a}-C^{\lambda a} \partial_{\lambda} b^{\mu}\right)+\frac{1}{6} \varepsilon_{b c}\left[\left(C^{\sigma b} \partial_{\sigma} C^{\lambda a}+C^{\sigma a} \partial_{\sigma} C^{\lambda b}\right) \partial_{\lambda} C^{\mu c}\right.\right. \\
&\left.\left.-C^{\lambda c} \partial_{\lambda}\left(C^{\sigma b} \partial_{\sigma} C^{\mu a}+C^{\sigma a} \partial_{\sigma} C^{\mu b}\right)\right]\right) \\
& X_{3}^{\mu a b}=-\varepsilon^{a b} b+\frac{1}{2}\left(\partial_{\mu} C^{b}\right) C^{\mu a}, \\
& X_{3}^{\mu a b}=-\varepsilon^{a b} b^{\mu}+\frac{1}{2}\left(C^{\lambda b} \partial_{\lambda} C^{\mu a}+C^{\lambda a} \partial_{\lambda} C^{\mu b}\right), \\
& Y_{1 \mu}^{i}= \varepsilon^{i j} b e_{\mu j}+b^{\lambda} \partial_{\lambda} e_{\mu}^{i}+e_{\lambda}^{i} \partial_{\mu} b^{\lambda}+\frac{1}{2} \varepsilon_{a b}\left(\left(e_{\mu}^{i} C^{b}+\varepsilon^{i j} C^{\lambda b} \partial_{\lambda} e_{\mu j}+\varepsilon^{i j} e_{\lambda j} \partial_{\mu} C^{\lambda b}\right) C^{a}\right. \\
&-C^{\lambda a} \partial_{\lambda}\left(\varepsilon^{i j} e_{\mu j} C^{b}+C^{\sigma b} \partial_{\sigma} e_{\mu}^{i}+e_{\sigma}^{i} \partial_{\mu} C^{\sigma b}\right) \\
&\left.+\left(\varepsilon^{i j} e_{\lambda j} C^{b}+\left(\partial_{\sigma} e_{\lambda}^{i}\right) C^{\sigma b}+e_{\sigma}^{i} \partial_{\lambda} C^{\sigma b}\right) \partial_{\mu} C^{\lambda a}\right), \\
&-\partial_{\mu} b+b^{\lambda} \partial_{\lambda} \omega_{\mu}+\omega_{\lambda} \partial_{\mu} b^{\lambda}-\frac{1}{2} \varepsilon_{a b}\left[C^{\lambda a} \partial_{\lambda}\left(C^{\sigma b} \partial_{\sigma} \omega_{\mu}+\omega_{\sigma} \partial_{\mu} C^{\sigma b}-\partial_{\mu} C^{b}\right)\right. \\
& Y_{1 \mu}=\left.\left.-2 X_{2}^{a b} \partial_{\sigma} \omega_{\lambda}+\omega_{\sigma} \partial_{\lambda} C^{\sigma b}-\partial_{\lambda} C^{b}\right) \partial_{\mu} C^{\lambda a}\right], \\
& Y_{2}^{a}=-2 X_{2}^{\mu a} . \\
& Y_{2}^{\mu a}=
\end{aligned}
$$

We choose the gauge boson $F=F(\phi)$ in the form ( $p, q$ are constant parameters)

$$
F=\int d^{2} x\left\{\frac{p}{2} \eta_{i j} \eta^{\mu \nu} e_{\mu}^{i} e_{\nu}^{j}+\frac{q}{2} \eta^{\mu \nu} \omega_{\mu} \omega_{\nu}\right\}
$$

Then, integrating in Eq. (18) over the variables $\lambda^{A}, \pi^{A a}, \bar{\phi}_{A}, \phi_{A a}^{*}$ and taking Eq. (21) into account, we have the following representation for $Z(J)$ :

$$
Z(J)=\int d \phi \exp \left\{\frac{i}{\hbar}\left(\mathcal{S}+S_{\mathrm{GF}}(\phi)+S_{\mathrm{GH}}(\phi)+J_{A} \phi^{A}\right)\right\}
$$

where

$$
\begin{aligned}
S_{\mathrm{GF}}= & \int d^{2} x\left(\chi b+\chi_{\mu} b^{\mu}\right), \\
S_{\mathrm{GH}}= & \int d^{2} x\left\{p \left(\eta^{\mu \nu}\left(e_{\lambda}^{i} \partial_{\nu} C^{\lambda a}-e_{\nu}^{i} \partial_{\lambda} C^{\lambda a}\right)\right.\right. \\
& \left.+\eta^{\lambda \nu} e_{\nu}^{i} \partial_{\lambda} C^{\mu a}\right)\left(\varepsilon_{i j} C^{b} e_{\mu}^{j}-\eta_{i j}\left(C^{\sigma b} \partial_{\sigma} e_{\mu}^{j}+e_{\sigma}^{j} \partial_{\mu} C^{\sigma b}\right)\right) \\
& -q\left(\eta^{\mu \nu}\left(\partial_{\nu} C^{a}-\omega_{\lambda} \partial_{\nu} C^{\lambda a}+\omega_{\nu} \partial_{\lambda} C^{\lambda a}\right)\right. \\
& \left.\left.-\eta^{\lambda \nu} \omega_{\nu} \partial_{\lambda} C^{\mu a}\right)\left(\partial_{\mu} C^{b}-C^{\sigma b} \partial_{\sigma} \omega_{\mu}-\omega_{\sigma} \partial_{\mu} C^{\sigma b}\right)\right\}
\end{aligned}
$$

and

$$
\begin{aligned}
\chi & =q \eta^{\mu \nu} \partial_{\mu} \omega_{\nu} \\
\chi_{\mu} & =\eta^{\lambda \nu}\left\{p\left(e_{\lambda i} \partial_{\mu} e_{\nu}^{i}-\partial_{\lambda}\left(e_{\mu i} e_{\nu}^{i}\right)\right)+q\left(\omega_{\lambda} \partial_{\mu} \omega_{\nu}-\partial_{\lambda}\left(\omega_{\mu} \omega_{\nu}\right)\right)\right\}
\end{aligned}
$$


We finally consider the initial theory (1), (3), (5), (6) in the framework of the background field method (see, for example, Ref. 25). For this purpose, we first assign to the initial fields the set $(A, Q)$ of the background $A=\left(e_{\mu}^{i}, \omega_{\mu}\right)$ and quantum $Q=\left(q_{\mu}^{i}, q_{\mu}\right)$ fields. Secondly, we associate the initial gauge transformations (3), (5) with two kinds of transformations, namely background $\delta_{B}$ and quantum $\delta_{Q}$, by the rule

$$
\begin{aligned}
\delta_{B} e_{\mu}^{i} & =\varepsilon^{i j} e_{\mu j} \zeta+e_{\nu}^{i} \partial_{\mu} \xi^{\nu}+\left(\partial_{\nu} e_{\mu}^{i}\right) \xi^{\nu}, \\
\delta_{B} \omega_{\mu} & =-\partial_{\mu} \zeta+\omega_{\nu} \partial_{\mu} \xi^{\nu}+\left(\partial_{\nu} \omega_{\mu}\right) \xi^{\nu}, \\
\delta_{B} q_{\mu}^{i} & =\varepsilon^{i j} q_{\mu j} \zeta+q_{\nu}^{i} \partial_{\mu} \xi^{\nu}+\left(\partial_{\nu} q_{\mu}^{a}\right) \xi^{\nu}, \\
\delta_{B} q_{\mu} & =q_{\nu} \partial_{\mu} \xi^{\nu}+\left(\partial_{\nu} q_{\mu}\right) \xi^{\nu}, \\
\delta_{Q} e_{\mu}^{i}= & \delta_{Q} \omega_{\mu}=0 \\
\delta_{Q} q_{\mu}^{i}= & \varepsilon^{i j}\left(e_{\mu j}+q_{\mu j}\right) \zeta+\left(e_{\nu}^{i}+q_{\nu}^{i}\right) \partial_{\mu} \xi^{\nu}+\left(\partial_{\nu} e_{\mu}^{i}+\partial_{\nu} q_{\mu}^{i}\right) \xi^{\nu}, \\
\delta_{Q} q_{\mu}=- & -\partial_{\mu} \zeta+\left(\omega_{\nu}+q_{\nu}\right) \partial_{\mu} \xi^{\nu}+\left(\partial_{\nu} \omega_{\mu}+\partial_{\nu} q_{\mu}\right) \xi^{\nu} .
\end{aligned}
$$

Clearly, the action $\mathcal{S}(A+Q)$ in Eq. (1) is invariant under both kinds of transformations (23), (24).

Following the background field method, we further introduce the analog $Z(J, A)$ of the generating functional of Green's functions (its relation to the standard generating functional has been established in Ref. 27)

$$
Z(J, A)=\int d Q d \bar{C} d C \exp \left\{\frac{i}{\hbar}\left(\mathcal{S}(A+Q)+S_{\mathrm{GF}}(A, Q)+S_{\mathrm{GH}}(A, Q ; \bar{C}, C)+J Q\right)\right\},
$$

where $J=\left(J_{i}^{\mu}, J^{\mu}\right)$ are the sources to the quantum fields $Q=\left(q_{\mu}^{i}, q_{\mu}\right)$. In Eq. (25), $S_{\mathrm{GH}}=$ $S_{\mathrm{GH}}(A, Q)$ is a functional constructed with the help of the gauge fixing functions $\chi, \chi_{\mu}$ (respectively for the parameters $\left.\zeta, \xi^{\mu}\right)$ according to the requirement of invariance under the background transformations, i.e. $\delta_{B} S_{\mathrm{GH}}=0$. Given this, the functional $S_{\mathrm{GH}}=S_{\mathrm{GH}}(A, Q ; \bar{C}, C)$ is constructed by the rule

$$
S_{\mathrm{GH}}=\int d^{2} x\left(\bar{C} \delta_{Q} \chi+\bar{C}^{\mu} \delta_{Q} \chi_{\mu}\right)
$$

where in the transformations $\delta_{Q}$ we make the replacement $\left(\zeta, \xi^{\mu}\right) \rightarrow\left(C, C^{\mu}\right)$.

Let us introduce the gauge-fixing conditions $\chi=\chi(A, Q), \chi_{\mu}=\chi_{\mu}(A, Q)$ linear in the quantum fields $Q=\left(q_{\mu}^{i}, q_{\mu}\right)$

$$
\begin{gathered}
\chi=e g^{\mu \nu} \nabla_{\mu} q_{\nu}, \\
\chi_{\mu}=e g^{\lambda \nu} e_{\mu i} \nabla_{\lambda} q_{\nu}^{i},
\end{gathered}
$$

where $e, g^{\mu \nu}$ are constructed from the background fields $e_{\mu}^{i}\left(g^{\mu \lambda} g_{\lambda \nu}=\delta_{\nu}^{\mu}, g_{\mu \nu}=\eta_{i j} e_{\mu}^{i} e_{\nu}^{j}\right.$, $e=\operatorname{det} e_{\mu}^{i}$ ), and the action of the covariant derivative $\nabla_{\mu}$ on an arbitrary (psedo-)tensor field $T_{\mu_{1} \ldots \mu_{k} i_{1} \ldots i_{m}}^{\nu_{1} \ldots \nu_{2} j_{1} \ldots j_{n}}$ is given by the rule $\left(\left(\Omega_{\mu}\right)_{j}^{i}=\varepsilon^{i k} \eta_{k j} \omega_{\mu}\right)$

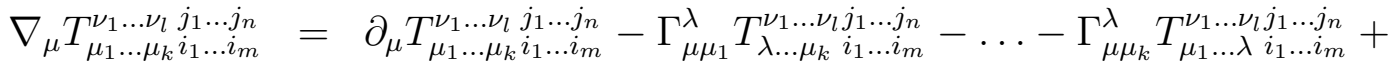

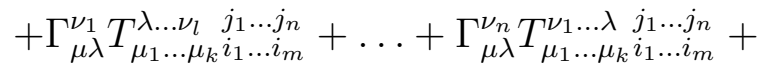

$$
\begin{aligned}
& +\left(\Omega_{\mu}\right)_{p}^{j_{1}} T_{\mu_{1} \ldots \mu_{k} i_{1} \ldots i_{m}}^{\nu_{1} \ldots \nu_{l}}+\ldots+\left(\Omega_{\mu}\right)_{p}^{j_{n}} T_{\mu_{1} \ldots \mu_{k} i_{1} \ldots i_{m}}^{\nu_{1} \ldots \nu_{l} j_{1} \ldots p}- \\
& -\left(\Omega_{\mu}\right)_{i_{1}}^{p} T_{\mu_{1} \ldots \mu_{k} p \ldots i_{m}}^{\nu_{1} \ldots \nu_{1} j_{1} \ldots j_{n}}-\ldots-\left(\Omega_{\mu}\right)_{i_{m}}^{p} T_{\mu_{1} \ldots \mu_{k} i_{1} \ldots p}^{\nu_{1} \ldots \nu_{l} j_{1} \ldots j_{n}}
\end{aligned}
$$


In Eq. (28), $\Gamma_{\mu \nu}^{\lambda}$ are constructed with the help of the metric $g_{\mu \nu}$

$$
\Gamma_{\mu \nu}^{\lambda}=\frac{1}{2} g^{\lambda \sigma}\left(\partial_{\nu} g_{\mu \sigma}+\partial_{\mu} g_{\nu \sigma}-\partial_{\sigma} g_{\mu \nu}\right)
$$

Clearly, the covariant derivative $\nabla_{\mu}(28)$ satisfies the property

$$
\nabla_{\mu}(F G)=F \nabla_{\mu} G+\left(\nabla_{\mu} F\right) G
$$

where $F, G$ are arbitrary (psedo-)tensor fields. At the same time, one readily establishes the relations

$$
\nabla_{\sigma} g_{\mu \nu}=\nabla_{\sigma} g^{\mu \nu}=0
$$

We choose for $S_{\mathrm{GF}}$ the functional ( $p, q$ are some numbers)

$$
S_{\mathrm{GF}}=\frac{1}{2} \int d^{2} x e\left(p \chi^{2}+q \chi_{\mu} \chi^{\mu}\right),
$$

invariant, by construction, under the local Lorentz transformations of the form

$$
\begin{aligned}
e_{\mu}^{i} & =\left(\Lambda e_{\mu}\right)^{i}, \quad q_{\mu}^{i}=\left(\Lambda q_{\mu}\right)^{i}, \\
\left(\Omega_{\mu}^{\prime}\right)_{b}^{i} & =\left(\Lambda \Omega_{\mu} \Lambda^{-1}\right)_{j}^{i}+\left(\Lambda \partial_{\mu} \Lambda^{-1}\right)_{j}^{i}, \quad q_{\mu}^{\prime}=q_{\mu},
\end{aligned}
$$

as well as under the general coordinates transformations

$$
\begin{aligned}
& e_{\mu}^{\prime i}\left(x^{\prime}\right)=\frac{\partial x^{\lambda}}{\partial x^{\prime \mu}} e_{\lambda}^{i}(x), \quad \omega_{\mu}^{\prime}\left(x^{\prime}\right)=\frac{\partial x^{\lambda}}{\partial x^{\prime \mu}} \omega_{\lambda}(x) \\
& q_{\mu}^{\prime i}\left(x^{\prime}\right)=\frac{\partial x^{\lambda}}{\partial x^{\prime} \mu} q_{\lambda}^{i}(x), \quad q_{\mu}^{\prime}\left(x^{\prime}\right)=\frac{\partial x^{\lambda}}{\partial x^{\prime} \mu} q_{\lambda}(x) .
\end{aligned}
$$

At the same time, the infinitesimal form of the transformations (30) and (31) coincides with the background transformations (23).

Notice that the (non-vanishing) quantum transformations (24) can be represented, with allowance for the definition (28), in the form $\left(\zeta \rightarrow C, \xi^{\mu} \rightarrow C^{\mu}\right)$

$$
\begin{aligned}
& \delta_{Q} q_{\mu}^{i}=\varepsilon^{i j}\left(e_{\mu j}+q_{\mu j}\right) C+\left(e_{\nu}^{i}+q_{\nu}^{i}\right) \nabla_{\mu} C^{\nu}+\left(\nabla_{\nu} e_{\mu}^{i}+\nabla_{\nu} q_{\mu}^{i}\right) C^{\nu}-\varepsilon^{i j} \omega_{\nu}\left(e_{\mu j}+q_{\mu b}\right) C^{\nu}, \\
& \delta_{Q} q_{\mu}=-\nabla_{\mu} C+\left(\omega_{\nu}+q_{\nu}\right) \nabla_{\mu} C^{\nu}+\left(\nabla_{\nu} \omega_{\mu}+\nabla_{\nu} q_{\mu}\right) C^{\nu} .
\end{aligned}
$$

Then the functional $S_{\mathrm{GH}}(26)$ takes on the form

$$
\begin{aligned}
S_{\mathrm{GH}}= & \int d^{2} x e\left\{-\bar{C} \nabla_{\mu} \nabla^{\mu} C+\bar{C} \nabla^{\mu}\left[\left(\nabla_{\nu} \omega_{\mu}+\nabla_{\nu} q_{\mu}\right) C^{\nu}+\left(\omega_{\nu}+q_{\nu}\right) \nabla_{\mu} C^{\nu}\right]+\right. \\
& +\varepsilon^{i j} \bar{C}^{\mu} e_{\mu i} \nabla^{\nu}\left[\left(e_{\nu j}+q_{\nu j}\right)\left(C-\omega_{\lambda} C^{\lambda}\right)\right]+ \\
& \left.+\bar{C}^{\mu} e_{\mu i} \nabla^{\nu}\left[\left(\nabla_{\lambda} e_{\nu}^{i}+\nabla_{\lambda} q_{\nu}^{i}\right) C^{\lambda}+\left(e_{\lambda}^{i}+q_{\lambda}^{i}\right) \nabla_{\nu} C^{\lambda}\right]\right\} .
\end{aligned}
$$

One readily establishes the fact that the quantum action $S=\mathcal{S}+S_{\mathrm{GF}}+S_{\mathrm{GH}}(1)$, (29), (32) is invariant under the complex of the background transformations (23) and the transformations of the ghost fields

$$
\begin{aligned}
\delta \bar{C} & =\left(\partial_{\mu} \bar{C}\right) \xi^{\mu}, \quad \delta C=-C^{\mu} \partial_{\mu} \zeta+\left(\partial_{\mu} C\right) \xi^{\mu} \\
\delta \bar{C}^{\mu} & =-\bar{C}^{\nu} \partial_{\nu} \xi^{\mu}+\left(\partial_{\nu} \bar{C}^{\mu}\right) \xi^{\nu} \\
\delta C^{\mu} & =-C^{\nu} \partial_{\nu} \xi^{\mu}+\left(\partial_{\nu} C^{\mu}\right) \xi^{\nu} .
\end{aligned}
$$


From Eqs. (23), (33) it follows immediately that the functional $Z(J, A)(25)$ is invariant under the complex of the initial gauge transformations (3), (5) of the background fields $A=\left(e_{\mu}^{i}, \omega_{\mu}\right)$ and the transformations of the sources $J=\left(J_{i}^{\mu}, J^{\mu}\right)$

$$
\begin{aligned}
& \delta J_{i}^{\mu}=\varepsilon_{i j} J^{\mu j} \zeta-J_{i}^{\nu} \partial_{\nu} \xi^{\mu}+\partial_{\nu}\left(J_{i}^{\mu} \xi^{\nu}\right), \\
& \delta J^{\mu}=-J^{\nu} \partial_{\nu} \xi^{\mu}+\partial_{\nu}\left(J^{\mu} \xi^{\nu}\right) .
\end{aligned}
$$

As a consequence of the invariance of $Z(J, A) \equiv \exp \left\{\frac{i}{\hbar} W(J, A)\right\}$ under (3), (5), (34) we have the invariance of the functional $\Gamma=\Gamma(\bar{Q}, A), \bar{Q}=\left(\bar{q}_{\mu}^{i}, \bar{q}_{\mu}\right)$

$$
\Gamma(\bar{Q}, A)=W(J, A)-J \bar{Q}, \quad \bar{Q}=\frac{\delta W}{\delta J}, \quad J=-\frac{\delta \Gamma}{\delta \bar{Q}}
$$

under the complex of the transformations (3), (5) and the transformations of the fields $\bar{Q}$

$$
\begin{aligned}
\delta \bar{q}_{\mu}^{i} & =\varepsilon^{i j} \bar{q}_{\mu j} \zeta+\bar{q}_{\nu}^{i} \partial_{\mu} \xi^{\nu}+\left(\partial_{\nu} \bar{q}_{\mu}^{i}\right) \xi^{\nu}, \\
\delta \bar{q}_{\mu} & =\bar{q}_{\nu} \partial_{\mu} \xi^{\nu}+\left(\partial_{\nu} \bar{q}_{\mu}\right) \xi^{\nu} .
\end{aligned}
$$

By virtue of Eq. (35), the effective action $\Gamma(A)$ defined in the background field method by the rule

$$
\Gamma(A)=\left.\Gamma(\bar{Q}, A)\right|_{\bar{Q}=0},
$$

is invariant under the initial gauge transformations (3), (5) of the background fields $A=$ $\left(e_{\mu}^{i}, \omega_{\mu}\right)$.

\section{Conclusion}

In this paper we have considered the model $^{7}$ of two-dimensional gravity with dynamical torsion (1), (3), (5) and performed its quantization using different Lagrangian methods. Thus, for the model in question we have obtained the generating functionals of Green's functions (17), (22) within the $\mathrm{BV}^{23}$ and $\mathrm{BLT}^{24}$ quantization formalisms for general gauge theories as well as the analog (25), (29), (32) of the generating functional of Green's functions applied for the construction of the gauge-invariant effective action in the background field method (see, for example, Ref. 25). Notice that, as far as the classically equivalent ${ }^{20}$ model (7), (11) with auxiliary fields is concerned, not only may it prove to be non-equivalent to (1), (3), (5) at the quantum level, but also its quantization should pose a considerable problem involving the solution of the generating equations for an open algebra of gauge transformations, as required (in contrast to the study of Ref. [20]) by the consistent quantization procedure. ${ }^{23,24}$

\section{Acknowledgments}

The authors are grateful to I. L. Buchbinder for useful discussions. The work has been partially supported by the Russian Foundation for Basic Research (RFBR), project 96-02-16017, as well as by the joint project of Deutsche Fortschungsgemeinschaft and Russian Foundation for Basic Research (DFG-RFBR), 96-02-00180G. 


\section{References}

1. R. Jackiw, Nucl. Phys. B252 (1985) 343.

2. C. Teitelboim, Phys. Lett. B126 (1983) P. 41; T. Banks and L. Susskind, Int. J. Theor. Phys. 23 (1984) 475; I. M. Lichtzier and S. D. Odintsov, Mod. Phys. Lett. A6 (1991) 1953.

3. N. Sanchez, Nucl. Phys. B266 (1986) 487.

4. J.D. Brown, M. Henneaux and C. Teitelboim, Phys. Rev. D33 (1986) 319.

5. R. Balbinot and R. Floreanini, Phys. Lett. B160 (1985) 401; R. Floreanini, Ann. Phys. (N. Y.) 167 (1986) 317.

6. T. Fukuyama and K. Kamimura, Phys. Lett. B160 (1985) 259.

7. M.O. Katanaev and I.V. Volovich, Ann. Phys. 197 (1990) 1.

8. M. Martellini, Ann. Phys. (N. Y.) 167 (1986) 437.

9. R. Marnelius, Nucl. Phys. B211 (1983) 14.

10. L. Brink, P. Di Vecchia and P. Howe, Phys. Lett. B65 (1976) 471; S. Deser and B. Zumino, Phys. Lett. B65 (1976) 369.

11. M.B. Green and J.D. Schwarz and E. Witten, Superstring Theory (Cambridge Univ. Press, London, 1987).

12. P. Van Nieuwenhuizen, Nucl. Phys. B211 (1983) 14.

13. M.O. Katanaev, J. Math. Phys. 31 (1991) 2483; 34 (1993) 22.

14. W. Kummer and D.J. Schwarz, Phys. Rev. 45 (1992) 3628.

15. S.N. Solodukhin, Int. J. Mod. Phys. D3 (1994) 269.

16. T. Strobl, Int. J. Mat. Phys. A8 (1993) 1383.

17. P. Schaller and T. Strobl, Class. Quant. Grav. 199411331.

18. F. Haider and W. Kummer, Int. J. Mod. Phys. A9 (1994) 207.

19. W. Kummer and D.J. Schwarz, Nucl. Phys. B382 (1992) 171.

20. N. Ikeda and K. Izawa, Prog. Theor. Phys. 89 (1993) 223.

21. M.O. Katanaev and I.V. Volovich, Phys. Lett. 175B (1986) 413.

22. P.M.Lavrov and I.V.Tyutin, Yad. Fiz. 50 (1989) 1467.

23. I.A. Batalin and G.A. Vilkovisky, Phys. Lett. 102B (1981) 27; Phys. Rev. D28 (1983) 2567.

24. I.A. Batalin, P.M. Lavrov and I.V. Tyutin, J. Math. Phys. 31 (1990) 1487; 32 (1991) $532 ; 32$ (1991) 2513. 
25. I.L. Buchbinder, S.D. Odintsov and I.L. Shapiro, Effective Action in Quantum Gravity (IOP Publ, Bristol and Philadelphia, 1992).

26. B.S. De Witt, Dynamical Theory of Groups and Fields (Gordon and Breach, New York, 1965).

27. L. F. Abbott, Nucl. Phys. B185 (1981) 189. 